\title{
Making a Traditional Spine Surgery Clinic Telemedicine-Ready in the "New Normal" of Coronavirus Disease 2019
}

\author{
Ka-Po Gabriel Liu ${ }^{1}$, Wei Loong Barry Tan ${ }^{1}$, Wei Luen James Yip ${ }^{2,3}$, Jun-Hao Tan ${ }^{1}$, Hee-Kit Wong ${ }^{1}$ \\ ${ }^{1}$ University Orthopaedics, Hand and Reconstructive Microsurgery Cluster, National University Health System, Singapore \\ ${ }^{2}$ Department of Cardiology, National University Heart Centre, Singapore \\ ${ }^{3}$ Medical Informatics Office, National University Health System, Singapore
}

Study Design: An original article describing a comprehensive methodology for making a traditional spine surgery clinic telemedicineready in terms of logistical considerations and workflow.

Purpose: The aim of this study is to promote the use of telemedicine via videoconferencing to reduce human exposure to severe acute respiratory syndrome coronavirus 2 (SARS-CoV-2) and reduce the risk of coronavirus disease 2019 (COVID-19) transmission at outpatient clinics.

Overview of Literature: The COVID-19 pandemic is the biggest healthcare crisis in the 21st century. Until a vaccine is developed or herd immunity against SARS-CoV-2 is achieved, social distancing to avoid crowding is an important strategy to reduce disease transmission and resurgence. Telemedicine has already been applied in the field of orthopedics with encouraging results.

Methods: We reviewed the evidence behind telemedicine and described our clinical protocol, patient selection criteria, and workflow for telemedicine. We discussed a simple methodology to convert pre-existing traditional clinic resources into telemedicine tools, along with future challenges.

Results: Our methodology was successfully and easily applied in our clinical practice, with a streamlined workflow allowing our spine surgery service to implement telemedicine as a consultation modality in line with the national recommendations of social distancing.

Conclusions: Telemedicine was well incorporated into our outpatient practice using the above workflow. We believe that the use of telemedicine via videoconferencing can become part of the new normal and a safe strategy for healthcare systems as both a medical and an economic countermeasure against COVID-19.

Keywords: SARS-CoV-2; COVID-19; Telemedicine; Remote consultation; Videoconferencing; Spine surgery; Spine clinic

Received Oct 22, 2020; Revised Jan 30, 2021; Accepted Feb 17, 2021

Corresponding author: Ka-Po Gabriel Liu

University Orthopaedics, Hand and Reconstructive Microsurgery Cluster, National University Health System, 1E Kent Ridge Road, NUHS Tower Block Level 11, 119228, Singapore

Tel: +65-6772-5611, Fax: +65-6777-3295, E-mail: Gabriel_liu@nuhs.edu.sg 


\section{Introduction}

\section{The rationale for the rapid adoption of telemedicine}

The coronavirus disease 2019 (COVID-19) pandemic has developed into the greatest crisis in the modern world since its emergence from Wuhan in December 2019 [1]. As of October 3rd, 2020, the number of confirmed cases has surpassed 34 million, with more than 1,026,000 deaths [2]. Based on multiple epidemiological studies and simulation models, the key to successfully containing the spread of severe acute respiratory syndrome coronavirus 2 (SARS-CoV-2: COVID-19) is early detection, early isolation, and early intervention by means of social distancing and the reduction of non-essential human contact $[3,4]$. These steps are paramount for keeping the COVID-19 incidence curve flat until an effective vaccine is developed. Even after the curve has been flattened, avoiding a second or third wave of disease depends on the avoidance of unnecessary human exposure to SARS-CoV-2 in this new normal state, until herd immunity against the virus has been obtained.

The aim of this paper is to call for the use of telemedicine to reduce human exposure to SARS-CoV-2 in an outpatient setting. We describe our university spine clinic protocol, established methodology, and workflow considerations for the easy and rapid adoption of telemedicine in this extraordinary time.

\section{Evidence supporting telemedicine in spinal surgery}

Telemedicine has already been applied in the field of orthopedics with encouraging results. In the year 2000, Haukipuro et al. [5] performed the first orthopedic telemedicine randomized controlled trial (RCT) in Finland and found that, despite the inability to perform physical examinations, patients were satisfied with the videoconferencing nature of the consultation. In addition, Harno et al. [6] showed a $45 \%$ cost saving in orthopedic telemedicine consultations compared with traditional faceto-face consultations. Moreover, in 2019, Buvik et al. [7,8] reported two RCTs of telemedicine in remote orthopedic consultations. The studies found no difference in patientreported satisfaction and health outcomes between videoassisted and standard consultations, with a significantly high proportion of patients being keen to continue with video-assisted consultations for their repeat consultation.
Sultan et al. [9] studied 189 telemedicine virtual visits for pediatric patients with spinal deformity and found that the patients who underwent telemedicine consultations received faster care, with shorter waiting times to appointments and high patient satisfaction scores comparable to those of standard visits. Similar positive results with telemedicine have been reported in patients suffering spinal cord injuries [10] and chronic spinal pain [11].

In the era of the COVID-19 pandemic, reducing patient density in clinics by adopting telemedicine via videoconferencing is an important strategy that must be deployed as a matter of urgency. It has been reported that, among those infected with SARS-CoV-2, the asymptomatic carrier rate is up to $80 \%[12,13]$, with a prolonged period of viral shedding via the respiratory and fecal routes for up to 37 and 47 days, respectively, after the onset of symptoms $[12,13]$. These concerns add extra weight to the need to reduce patient density in clinics.

The additional advantages of telemedicine use during the COVID-19 pandemic include the following [14-16]: (1) providing continued medical care to patients whose condition is not deemed to be medically urgent for elective hospital admission; (2) protecting healthcare workers and patients from the risk of SARS-CoV-2 exposure and transmission; (3) reducing patient backlog during social distancing measures; (4) reducing demands on administrative staff for continuous rescheduling of appointments via contacting patients and re-bookings on the system during periods of partial or complete lockdown; (5) maintaining financial viability of outpatient practice to cover hospital overhead costs.

\section{Materials and Methods}

Patient selection is the key to success in telemedicine consultation (Table 1). We prioritize follow-up cases for imaging (magnetic resonance imaging [MRI]) review, postoperative follow-up, and long-term annual follow-ups as the first group of patients. In these patients, we do not expect any significant deterioration in their neurological status in most cases. The MRI-review patients would already have been assessed recently in the clinic, and the postoperative patients would have been examined during the course of their hospital stay and upon discharge. Annual review patients are generally stable and can be assessed by teleconsultations, with the option of bringing them in for a physical consultation should their symp- 
Table 1. Patient selection criteria for telemedicine via videoconferencing

\section{Patients for:}

Review new investigation results (e.g., X-ray, MRI, nerve conduction

1 study): typically, a 2nd consultation after index traditional clinic consult ${ }^{\mathrm{a})}$

2 Interval review for postoperative follow-up ${ }^{\text {al }}$

3 Yearly patient follow-upa)

4 Preliminary discussion of surgical options

5 Patients who were previously investigated with MRI but was lost to follow-up

6 Adolescent idiopathic scoliosis follow-up ${ }^{\text {al }}$

7 Immediate postoperative wound care

New case not reviewed in clinic before (1) with investigations performed by general practitioners and (2) no prior investigations at all

$\mathrm{MRI}$, magnetic resonance imaging

alPatient performs radiological investigation 1 week before attending telemedicine consultation

toms change. Patients with known underlying pathology requesting a preliminary surgical discussion or previously seen patients with similar recurrent complaints who were previously investigated but lost to follow-up can be scheduled for telemedicine consultations as well.

The second group of patients would be those requiring a follow-up review for adolescent idiopathic scoliosis. These patients are generally stable without neurological deficits and only require simple observation, brace therapy, or postoperative review.

The last group of patients that we are exploring at this point would be those requiring wound review and a new or first-visit patient consultation. Patients immediately after an operation or those with delayed wound complications that require weekly to twice weekly wound reviews may undergo telemedicine consultations. The actual dressing changes can be performed at home by the patients themselves or competent caregivers.

\section{Results}

\section{Conversion of pre-existing traditional clinic aids into telemedicine tools}

The construction of a comprehensive, customized telemedicine videoconferencing platform may take a long time and be expensive. A few quick and simple steps can be used to convert traditional clinic aids into telemedicine tools, as shown in Table 2. One of the difficulties with the use of telemedicine is confirmation of patients' symptoms.
Table 2. Simple way to convert traditional clinical aids into telemedicine tools

$\begin{array}{lll}\text { Clinic aids } & \begin{array}{c}\text { Conversion } \\ \text { methodology }\end{array} \\ \text { rior and posterior views) on } \\ \text { which surgeon can draw } \\ \text { to confirm the location of } \\ \text { symptoms (red for pain, } \\ \text { blue for numbness, and } \\ \text { green for paresthesia) }\end{array}$

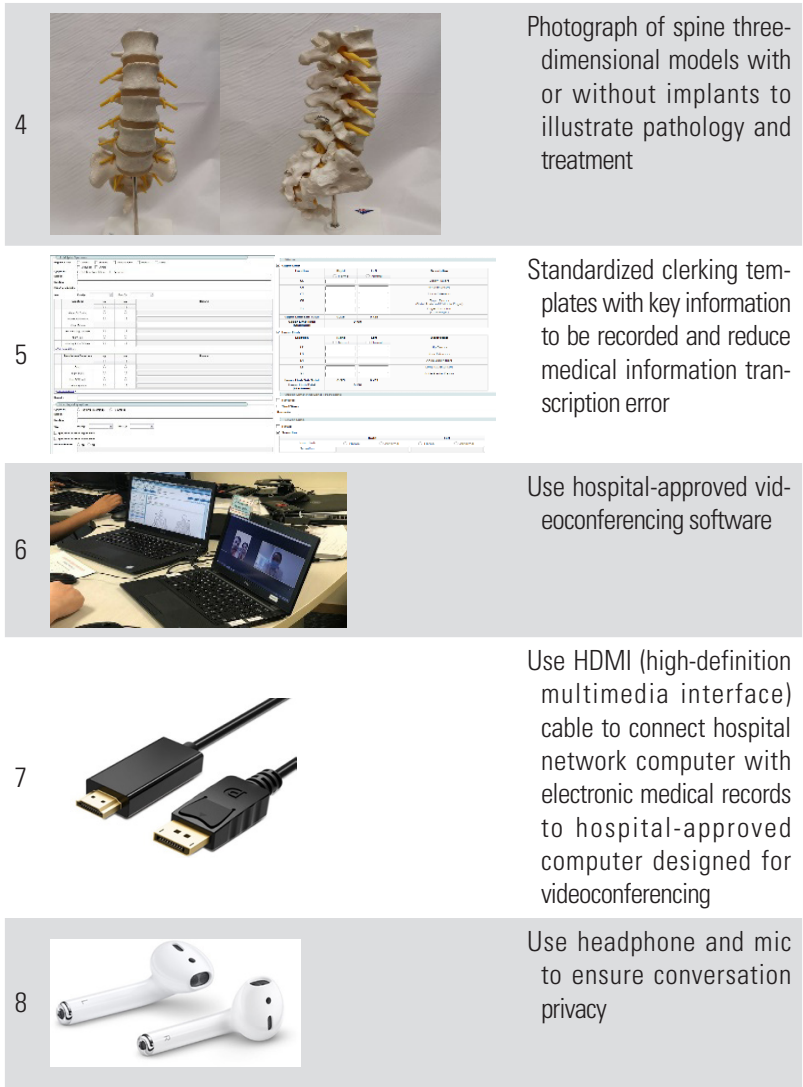

This is especially so if the patient complains of back pain and sciatica, as the videoconferencing display only captures the face and upper torso of the patient, and it would be difficult for the patient to show the exact location of the pain on his or her back and legs in order for the surgeon to locate the dermatome of the nerve root pathology. 


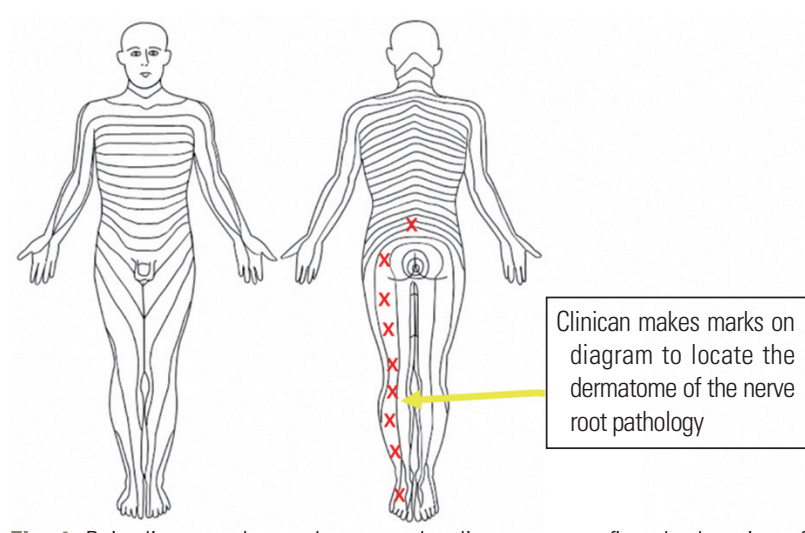

Fig. 1. Pain diagram: doctor draws on the diagram to confirm the location of symptoms in the patient (red: pain, blue: numbness, green: paresthesia).

To facilitate this, we scan a clinical body diagram with anterior and posterior views that can be charted by the clinician after ascertaining the symptoms of the patient (Fig. 1), and can be shown to the patient for telemedicine confirmation on the computer via the share-screen function on video conferencing software (Fig. 2). Different colors are used to illustrate symptoms (e.g., red for pain, blue for numbness, and green for paresthesia). The diagrams are later scanned as electronic records into our patient records system. In our hospital, this body diagram is part of our electronic history and physical examination template for patient symptoms. The use of a standardized electronic template during video conferencing can further reduce errors occurring during the transcription of medical information.

Another simple step made to quantify the severity of the patient's symptoms is by scanning a copy of the Visual Analog Scale for pain as part of the telemedicine illustrative tool. This is shown on the shared screen during video consultation and allows the patient to effectively rank the severity of the symptoms.

In addition, we recommend scanning all pre-existing brochures for patient education and using spine models with and without implants available in the clinic as illustration tools during telemedicine consultation. These steps do not cost money and provide on-point information required for that specialty in the clinic. Information such as explanations of common pathologies (e.g., prolapsed intervertebral disc, scoliosis, and spinal stenosis), back care physiotherapy, and core strengthening exercises can be provided to patients during a videoconference and electronic copies of these reading materials can be emailed to them after the consultation. The use of these

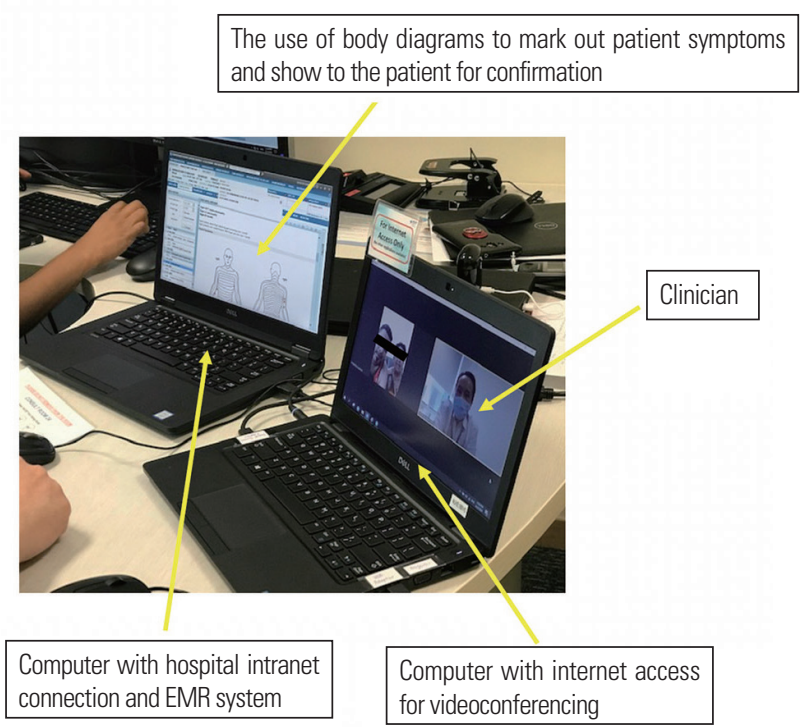

Fig. 2. Set up: computer with hospital intranet system with cable-link for screen projection to computer with internet access. EMR, electronic medical records.

brochures, which are often written by and familiar to clinic specialists, may aid the surgeon in giving a better illustration during consultation and shortening clinicians' telemedicine proficiency learning curve.

In our hospital, like many others, to maintain strict cybersecurity, the majority of hospital network computers do not have internet access. To circumvent this, an HDMI (high-definition multimedia interface) cable is used to connect the hospital network computer with the electronic medical records (EMR) system to a hospital-approved laptop with a webcam and internet access for videoconferencing. The operator should be trained to ensure cybersecurity and protect patient privacy. Headphones with a microphone are recommended to ensure privacy of communication and to avoid shouting at the patient in a noisy clinical environment due to poor internet reception. We advise consulting the hospital's information technology (IT) department and using only hospital-approved videoconferencing software (e.g., Zoom Pro [Zoom Video Communications Inc., San Jose, CA, USA] or Microsoft Teams [Microsoft Corp., Redmond, WA, USA]).

\section{Workflow for teleconsultations}

1) Patient scheduling

To maximize the success of telemedicine via videoconferencing, a dedicated telemedicine clinic coordinator is important. Weeks prior to telemedicine consultations, the 
clinic coordinator will show the patient list scheduled for traditional face-to-face clinic review to the surgeon, in order to identify suitable candidates for the telemedicine service. Using the above-mentioned guidelines, patients who have a good history with clinical pathologies that do not require detailed physical examinations are selected. At this point, we limit our teleconsultations to only review cases, as first-visit cases require a baseline physical examination for accurate diagnosis and these patients would benefit more from traditional face-to-face consultations to mitigate potential misdiagnosis. If investigations such as using radiographs or MRIs are required prior to the telemedicine consultation, these are performed 1 to 2 weeks prior to the consultation at a scheduled time, where the patient undergoes the investigation and can return home immediately thereafter, to avoid a prolonged stay in the hospital due to waiting for the traditional face-to-face consultation, making payment, and collecting medication.

The patients for whom teleconsultation is suitable are then contacted via phone or email to determine whether they are willing to participate in teleconsultation. Our University Spine Center's hotline and email address are provided should the patient require further verification. If the reply is positive, the clinic coordinator will email the patient with standardized instructions to set up the teleconsultation platform and follow-up with a phone call to address any queries and finalize the appointment time. The email will include the secured teleconsultation platform invite link (password-protected, with the password provided in a separate email) and a checkbox to obtain informed consent for the teleconsultation [17-19]. The coordinator will also inform the patient of any radiology appointment, and to advise them that should they experience symptoms such as fever, shortness of breath, or cough on the day of the radiology appointment, to call the clinic hotline and their appointment will be deferred. This reduces the risk of the patient transmitting infection to those in the healthcare facility.

2) The day of telemedicine consultation via video conferencing

It is recommended that the surgeon review all imaging reports by the radiologist prior to the sessions of teleconsultation, to guide the consultation process and avoid missing critical incidental findings, such as an unexpected renal mass discovered on MRI of the spine. The clinic staff then set up the teleconsultation platform, and the surgeon will be called in once the patient is online. At the very start of the teleconsultation, the surgeon reads a standardized disclaimer that the consultation will be recorded for clinical purposes, there should be no recording by the patient, and that the teleconsultation does not include a physical examination and hence may not be as holistic as a face-to-face consultation, which may be required to guide the final treatment plans. The patient is then required to identify him- or herself by displaying on-screen his or her National Identity Card or passport with their photograph for verification. The address of the participating patient is confirmed on-screen to provide the necessary location for sending an ambulance service should the patient collapse during the consultation.

The surgeon then takes a history from the patient according to the standardized electronic spine template, and utilizes the clinical diagrams, models, and brochures where applicable. Imaging investigations can be reviewed with the patient via projection onto the patient interview screen on the videoconferencing computer. The clinic staff will ensure that the required orders are carried out, such as physiotherapy appointments, delivery of prescribed medication, and billing of consultation charges.

The clinical information obtained during telemedicine consultation is transcribed onto the EMR system by the surgeon or junior medical staff. Once the teleconsultation details have been transcribed, the telemedicine recording is deleted (Fig. 3).

\section{Different workflow during partial lockdown and the "new normal" state}

During partial lockdown where the spine surgery division is segregated into separate teams, this telemedicine consultation platform is also set up in the office block where surgeons who are not scheduled in the clinic block can also review patients. After the COVID-19 curve is flattened, and in the new normal state, we expect that telemedicine consultation will remain and be integrated as part of the service provided during our normal clinic sessions. The clinic will provide two to three rooms for consultation, and approximately $50 \%$ of the booked patients (e.g., first-visit cases) will experience a traditional faceto-face consultation with physical examination, while the remaining $50 \%$ of patients in the same clinic session will be consulted via telemedicine videoconferencing to avoid overcrowding of the clinic. 
After curve flattens or new normal

state reached, 1-2 rooms for

face-to-face consultation and

1 room for telemedicine

\section{Clinic presents to surgeon the patient list for the scheduled session}

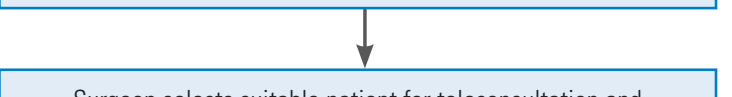

Surgeon selects suitable patient for teleconsultation and determines if prior imaging is required

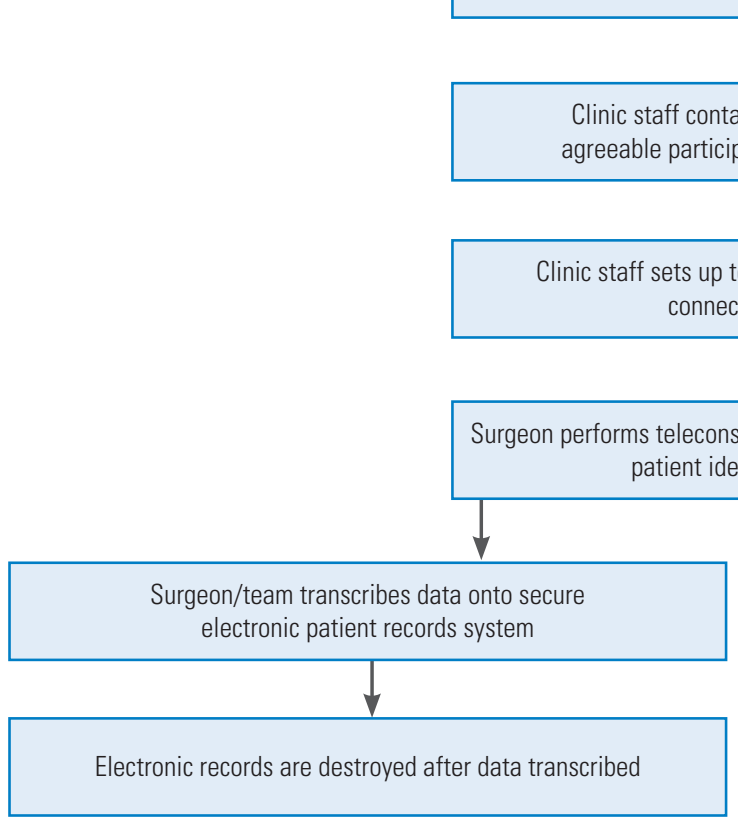

Schedules next appointment (teleconsult or face-to-face) and/or schedules procedures such as further investigations or surgery
Patient attends radiology appointment for imaging investigation
Surgeon reviews imaging results and reports before teleconsultation

Fig. 3. Workflow for telemedicine via video-consultation.

\section{Discussion}

\section{Challenges of teleconsultation in spinal surgery}

There are several challenges that may be encountered during the implementation of teleconsultations.

\section{1) Hospital network capability}

As a significant number of clinicians in the hospital may adopt telemedicine, the network capabilities of the hospital servers will require assessment and possible expansion before implementation, to avoid overloading the hospital network servers.

\section{2) Internet security}

The cybersecurity risk presented by using a virtual platform to discuss patient clinical data has to be regarded as a key concern for implementation. The security concerns in utilizing videoconferencing platforms have been discussed extensively, particularly in the COVID-19 pandemic era of social distancing, where several platforms have come under scrutiny after an exponential increase in their use [20]. Close cooperation with the hospital IT security department should mitigate these risks and allow the telemedicine team to comply with the laws and regulations set by the government.

\section{3) Medicolegal implications}

Teleconsultation is still in its infancy and has not yet been subject to medicolegal scrutiny. It is imperative that the surgeon discusses the potential limitations in telemedicine and has a joint decision-making process to optimize patient management. A detailed and clear record of the consultation history, review of investigations, discussion, and management in the EMR are of paramount importance.

\section{Limitations of telemedicine in elderly patients who are not technologically literate, or patients who do not have access to computers}

Teleconsultations require a patient with a clinical condition suitable to be followed up in a virtual setting, and ba- 
sic knowledge of and access to teleconferencing platforms. Some elderly patients may not be technologically literate, while others may not have access to computers. However, those with access to a phone that can support teleconferencing applications can still participate in telemedicine. For elderly patients who are not technologically literate, a younger, more technologically savvy family member can assist in the set up.

\section{Conclusions}

As humanity faces the immense challenges posed by the COVID-19 pandemic, it is necessary for the medical fraternity to revolutionize its clinical practices to fulfill its obligations, maintain social distancing to avoid SARSCoV-2 viral exposure, and preserve healthcare resources. In this article, we have described detailed patient selection criteria, implementation workflow, and simple steps to convert pre-existing traditional clinical resources into telemedicine tools to ensure telemedicine success. Embracing the technological advancements in information technology and applying them to the process of consultation with telemedicine via video conferencing allows clinicians and healthcare systems to adopt a safe strategy as both medi$\mathrm{cal}$ and economic countermeasures against COVID-19.

\section{Conflict of Interest}

No potential conflict of interest relevant to this article was reported.

\section{Author Contributions}

All authors participated in the concept of the manuscript and the preparation of the manuscript.

\section{References}

1. BBC News. Coronavirus: worldwide death toll climbs to 200,000. BBC News [Internet]. 2020 Apr 25 [cited 2021 Oct 2]. Available from: https://www.bbc.com/ news/world-52428162.

2. Johns Hopkins Coronavirus Resource Center. COVID-19 dashboard [Internet]. Baltimore (MD): Johns Hopkins Coronavirus Resource Center; 2020 [cited 2021 Oct 2]. Available from: https://coronavirus.jhu. edu/map.html.
3. Hellewell J, Abbott S, Gimma A, et al. Feasibility of controlling COVID-19 outbreaks by isolation of cases and contacts. Lancet Glob Health 2020;8:e48896.

4. Prem K, Liu Y, Russell TW, et al. The effect of control strategies to reduce social mixing on outcomes of the COVID-19 epidemic in Wuhan, China: a modelling study. Lancet Public Health 2020;5:e261-70.

5. Haukipuro K, Ohinmaa A, Winblad I, Linden T, Vuolio S. The feasibility of telemedicine for orthopaedic outpatient clinics: a randomized controlled trial. J Telemed Telecare 2000;6:193-8.

6. Harno K, Arajarvi E, Paavola T, Carlson C, Viikinkoski P. Clinical effectiveness and cost analysis of patient referral by videoconferencing in orthopaedics. J Telemed Telecare 2001;7:219-25.

7. Buvik A, Bugge E, Knutsen G, Smabrekke A, Wilsgaard T. Patient reported outcomes with remote orthopaedic consultations by telemedicine: a randomized controlled trial. J Telemed Telecare 2019;25:4519.

8. Buvik A, Bergmo TS, Bugge E, Smaabrekke A, Wilsgaard T, Olsen JA. Cost-effectiveness of telemedicine in remote orthopedic consultations: randomized controlled trial. J Med Internet Res 2019;21:e11330.

9. Sultan AA, Acuna AJ, Samuel LT, et al. Utilization of telemedicine virtual visits in pediatric spinal deformity patients: a comparison of feasibility and patient satisfaction at a large academic center. J Pediatr Orthop 2020;40:e712-5.

10. Dallolio L, Menarini M, China S, et al. Functional and clinical outcomes of telemedicine in patients with spinal cord injury. Arch Phys Med Rehabil 2008;89:2332-41.

11. Cottrell MA, Hill AJ, O'Leary SP, Raymer ME, Russell TG. Clinicians' perspectives of a novel home-based multidisciplinary telehealth service for patients with chronic spinal pain. Int J Telerehabil 2018;10:81-8.

12. Zhou F, Yu T, Du R, et al. Clinical course and risk factors for mortality of adult inpatients with COVID-19 in Wuhan, China: a retrospective cohort study. Lancet 2020;395:1054-62.

13. Wu Y, Guo C, Tang L, et al. Prolonged presence of SARS-CoV-2 viral RNA in faecal samples. Lancet Gastroenterol Hepatol 2020;5:434-5.

14. Mann DM, Chen J, Chunara R, Testa PA, Nov O. COVID-19 transforms health care through telemedi- 
cine: evidence from the field. J Am Med Inform Assoc 2020;27:1132-5.

15. Hong $\mathrm{Z}$, Li N, Li D, et al. Telemedicine during the COVID-19 pandemic: experiences from Western China. J Med Internet Res 2020;22:e19577.

16. Ohannessian R, Duong TA, Odone A. Global telemedicine implementation and integration within health systems to fight the COVID-19 pandemic: a call to action. JMIR Public Health Surveill 2020;6:e18810.

17. Hall DE, Prochazka AV, Fink AS. Informed consent for clinical treatment. CMAJ 2012;184:533-40.

18. Desch K, Li J, Kim S, et al. Analysis of informed consent document utilization in a minimal-risk genetic study. Ann Intern Med 2011;155:316-22.
19. Kane B, Sands DZ. Guidelines for the clinical use of electronic mail with patients. The AMIA Internet Working Group, Task Force on Guidelines for the Use of Clinic-Patient Electronic Mail. J Am Med Inform Assoc 1998;5:104-11.

20. Hodge R. Zoom security issues: Zoom buys security company, aims for end-to-end encryption. CNET [Internet]. 2020 May 8 [cited 2021 Oct 2]. Available from: https://www.cnet.com/news/zoom-securityissues-zoombombings-continue-include-racistlanguage-and-child-abuse/. 